\title{
Current evidence for central analgesic effects of NSAIDs: an overview of the literature
}

\author{
Vuilleumier, Pascal H ; Schliessbach, Juerg ; Curatolo, Michele
}

\begin{abstract}
Non-steroidal anti-inflammatory drugs (NSAIDs) are widely prescribed for a variety of painful conditions. Their peripheral anti-inflammatory effect due to inhibition of prostaglandin synthesis is well documented. In the late 1980's, animal data suggested for the first time that NSAIDs might have central effects as well. Since that time, central inflammatory and nociceptive pathways that are potential targets of NSAIDs have been extensively studied in both animal and human models. This review provides an overview of the relevant literature implicated in the central effects of NSAIDs. The role of different enzymes and mediators, as well as the central effects of NSAIDs are discussed. Literature search was performed by PubMed NCBI. A large body of evidence supports the central effects of NSAIDs in animal models of inflammatory pain conditions. Relevant mechanisms that underlie this central action involve spinal upregulation of the enzyme cyclooxygenase, increased spinal prostaglandin E2 production, modulation of inhibitory fast synaptic currents in lamina I and II of the dorsal horn, and glycine-dependent modulation of pain. Results from animal models are not yet sufficiently supported by human studies. This does not necessarily imply that the central effects of NSAIDs are irrelevant to human pain, but rather that methodological and regulatory barriers are the limiting step to translating findings from animal studies to human research protocols.
\end{abstract}

DOI: https://doi.org/10.23736/S0375-9393.18.12607-1

Posted at the Zurich Open Repository and Archive, University of Zurich

ZORA URL: https://doi.org/10.5167/uzh-158371

Journal Article

Published Version

Originally published at:

Vuilleumier, Pascal H; Schliessbach, Juerg; Curatolo, Michele (2018). Current evidence for central analgesic effects of NSAIDs: an overview of the literature. Minerva Anestesiologica, 84(7):865-870.

DOI: https://doi.org/10.23736/S0375-9393.18.12607-1 


\title{
Current evidence for central analgesic effects of NSAIDs: an overview of the literature
}

\author{
Pascal H. VUILLEUMIER ${ }^{1}{ }^{*}$, Juerg SCHLIESSBACH ${ }^{2}$, Michele CURATOLO ${ }^{3}$
}

\begin{abstract}
1Department of Anesthesiology and Pain Medicine, Bern University Hospital, Inselspital, Bern, Switzerland; 2Institute of Anesthesiology, University Hospital Zurich, Zürich, Switzerland; ${ }^{3}$ Department of Anesthesiology and Pain Medicine, University of Washington, Seattle, WA, USA

*Corresponding author: Pascal H. Vuilleumier, Department of Anesthesiology and Pain Medicine, Bern University Hospital, Inselspital Bern, 3010 Bern, Switzerland. E-mail: pascal.vuilleumier@insel.ch
\end{abstract}

\begin{abstract}
A B S T R A C T
Non-steroidal anti-inflammatory drugs (NSAIDs) are widely prescribed for a variety of painful conditions. Their peripheral anti-inflammatory effect due to inhibition of prostaglandin synthesis is well documented. In the late 1980's, animal data suggested for the first time that NSAIDs might have central effects as well. Since that time, central inflammatory and nociceptive pathways that are potential targets of NSAIDs have been extensively studied in both animal and human models. This review provides an overview of the relevant literature implicated in the central effects of NSAIDs. The role of different enzymes and mediators, as well as the central effects of NSAIDs are discussed. Literature search was performed by PubMed NCBI. A large body of evidence supports the central effects of NSAIDs in animal models of inflammatory pain conditions. Relevant mechanisms that underlie this central action involve spinal upregulation of the enzyme cyclooxygenase, increased spinal prostaglandin $\mathrm{E}_{2}$ production, modulation of inhibitory fast synaptic currents in lamina I and II of the dorsal horn, and glycine-dependent modulation of pain. Results from animal models are not yet sufficiently supported by human studies. This does not necessarily imply that the central effects of NSAIDs are irrelevant to human pain, but rather that methodological and regulatory barriers are the limiting step to translating findings from animal studies to human research protocols.
\end{abstract}

(Cite this article as: Vuilleumier PH, Schliessbach J, Curatolo M. Current evidence for central analgesic effects of NSAIDs: an overview of the literature. Minerva Anestesiol 2018;84:865-70. DOI: 10.23736/S0375-9393.18.12607-1)

KEY WORDS: Non-steroidal anti-inflammatory agents - Prostaglandins - Cyclooxygenase 1 - Cyclooxygenase 2.

$\mathrm{W}$ orldwide, non-steroidal anti-inflammatory drugs (NSAIDs) are the backbone in first-line pain management. ${ }^{1}$ Hereby NSAIDs analgesic efficacy has been mainly explained by their peripheral effect in the setting of an inflammatory response to injury. Tissue damage is associated with the release of inflammatory mediators, leading to sensitization of peripheral nociceptors and thus causing sustained pain and hypersensitivity. Inhibition of the cyclooxygenase 1 and 2 (COX-1 and COX-2) by NSAIDs reduces the inflammatory response by inhibiting prostaglandin synthesis, thereby alleviating pain. Coxibs, the COX-2 selective inhibitors, were designed to reduce gastrointestinal side effects associated with conventional NSAIDs. In the early 1990's, evidence suggested for the first time that NSAIDs might exert an effect in the central nervous system, as intrathecally administered NSAIDs were able to abolish hyperalgesia caused by spinal excitatory neurotransmitters. ${ }^{2,} 3$ This review gives an overview of the most important experimental findings supporting a central mode of action of NSAIDs, starting with data from animal studies and linking them to possible mechanisms in humans. 


\section{COPYRIGHT $^{\odot} 2018$ EDIZIONI MINERVA MEDICA}

\section{Overview of mediators and mechanisms}

Two isoenzymes of cyclooxygenase exist, COX1 and COX-2, both of which are inhibited by traditional, i.e. nonselective, NSAIDs. Newer compounds called Coxibs selectively inhibit COX-2. COX-1 is constitutively expressed in peripheral tissues such as gastric mucosa, kidneys or blood platelets, whereas COX-2 is induced in various tissues during inflammatory processes. However, in the central nervous system, both isoforms are usually present. ${ }^{4}$ Upon nociceptive stimulation, COX catalyzes the rate-limiting step in prostaglandin synthesis by forming $\mathrm{PGH}_{2}$ from arachidonic acid. $\mathrm{PGH}_{2}$ is subsequently transformed to various isoforms, such as $\mathrm{PGD}_{2}, \mathrm{PGE}_{2}, \mathrm{PGF}_{2}$ and $\mathrm{PGI}_{2}$ (prostacyclin). In terms of pain and nociception, $\mathrm{PGE}_{2}$ is the most extensively studied. Prostaglandins exert their effects by binding to specific receptors DP, EP, FP and IP for $\mathrm{PGD}_{2}, \mathrm{PGE}_{2}, \mathrm{PGF}_{2}$ and $\mathrm{PGI}_{2}$, respectively. All of them are G-protein-coupled receptors that affect intracellular signaling by second messengers such as cAMP or inositol-triphosphate. ${ }^{5}$ Four receptor subtypes for $\mathrm{PGE}_{2}$ (EP1-EP4 receptors), with partially opposing signaling pathways, are responding to the naturally occurring agonist $\mathrm{PGE}_{2} \cdot{ }^{6}$ They have been described by Coleman et al. in 1994. ${ }^{7}$ Activation of EP1 receptors increase intracellular $\mathrm{Ca}^{2+}$, while EP2 and EP4 lead to increased cAMP. Effects of EP3 receptors seem to be mediated by a decrease in cAMP. ${ }^{8}$

\section{Spinal prostaglandins and COX inhibitors in animal models}

Among the different prostanoids, prostaglandin $\mathrm{E}_{2}\left(\mathrm{PGE}_{2}\right)$ was revealed to be a main contributor to painful responses in inflammatory conditions. ${ }^{9}$ As an example, intrathecal injection of $\mathrm{PGE}_{2}$ causes dose-dependent hyperalgesia, whereas $\mathrm{PGD}_{2}, \mathrm{PGF}_{2}$ and $\mathrm{PGI}_{2}$ have no such effect. ${ }^{10,11}$ Animal data revealed significant increases in spinal cord $\mathrm{PGE}_{2}$ levels in various pain models, such as peripheral inflammation ${ }^{12}$ and rat models of surgical ${ }^{13}$ and neuropathic pain. ${ }^{14}$ Whether this increase in $\mathrm{PGE}_{2}$ is due to COX-1 or COX-2 seems to depend on the nature of the painful stimulus: while inflammatory pain (e.g.

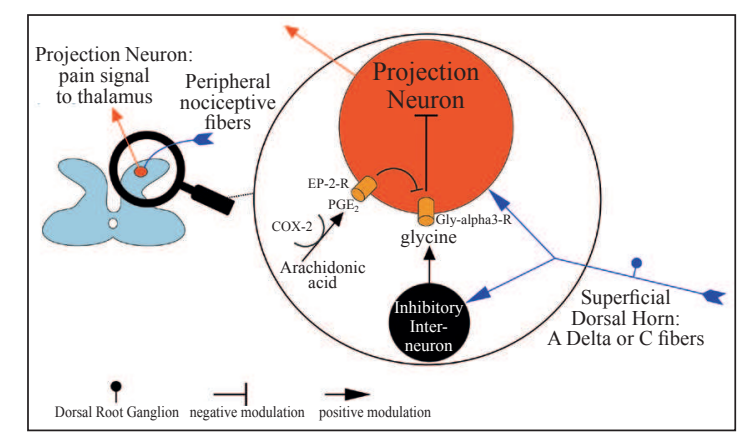

Figure 1.-Central effects of NSAIDs.

COX: cyclooxygenase; EP-2-R: EP2-receptor; $\mathrm{PGE}_{2}$ : prostaglandin E2; Gly-alpha3-R: glycine receptor $\alpha 3$.

injection of formalin or complete Freund's adjuvant) seems to upregulate spinal COX-2,15, 16 surgical pain by paw incision results in upregulation of spinal COX-1.17 The link between spinal $\mathrm{PGE}_{2}$ upregulation and pain could be confirmed experimentally in inflammatory pain models, whereby intrathecally administered inhibitors of COX-1 and COX-2 decreased spinal PGE $_{2}$ production with an associated decrease in central sensitization of pain. ${ }^{11,16,18,19}$ Insofar PGE $_{2}$-induced central sensitization seems to be mediated by a COX-2-PGE 2 response to proinflammatory cytokines, ${ }^{20-22}$ resulting in phosphorylation and inhibition of the glycine receptor $\alpha 3$ in the superficial spinal cord dorsal horn ${ }^{23,} 24$ (Figure 1). This was recently confirmed in a murine model of inflammatory pain, where 2,6-di-tert-buthylphenol reversed inflammation-mediated spinal nociception trough specific interaction with the phosphorylated glycine $\alpha 3$ receptors, thereby reducing hyperalgesia. ${ }^{25}$

\section{Role of EP receptors in animal pain}

The role of different EP receptor subtypes has been only partially clarified. Olivia et al. injected the non-selective prostaglandin agonist misoprostol into the periaqueductal grey (PAG) in mice. Subsequent application of specific agonists for EP1-4 receptor subtypes into the PAG all inhibited the central late phase of formalin-induced hyperalgesia. ${ }^{26}$ Nakayama et al. applied $\mathrm{PGE}_{2}$ intrathecally in rats and observed stable hyperalgesia associated with increased $\mathrm{Ca}^{2+}$ in the dorsal horn, which is the second messenger of EP1 receptors. 
Subsequent blockade of EP1 receptors normalized both hyperalgesia and $\mathrm{Ca}^{2+}$ levels, suggesting that $\mathrm{PGE}_{2}$ acts via EP1 receptors. ${ }^{27}$ Reinold et al. performed several experiments with EP1-3 receptor-deficient mice, aiming to identify the mechanisms and the subtypes of EP-receptors involved in hyperalgesia. Intrathecal injection of $\mathrm{PGE}_{2}$ caused mechanical and thermal hyperalgesia that were similar in EP3 $\%$ and wild type (WT) mice. No hyperalgesia developed in EP2\% mice. $\mathrm{PGE}_{2}$-injection into the hind paw caused similar thermal sensitization in all mice, but mechanical sensitization was significantly reduced in EP2 \% mice. Local inflammation of the paw by zymosan A caused long-lasting hyperalgesia in WT and EP3-/- mice, while EP2 - - showed initial hyperalgesia that recovered within hours. Taken together, these findings suggest a crucial role of the EP2 receptor in spinal long-lasting hyperalgesia, and maybe a partial role of peripheral EP2 receptors in localized mechanical hyperalgesia. ${ }^{11}$

Johansson et al. investigated the contribution of EP1 receptors to inflammatory pain. ${ }^{28}$ With the same laboratory setup as Reinold et al., this time using EP1\%- mice, they showed significantly less hyperalgesia to heat stimuli after peripheral $\mathrm{PGE}_{2}$ injection compared to WT or EP2 /- mice, suggesting that EP1 receptors are responsible for peripheral rather than spinal sensitization. ${ }^{28}$

Intrathecal injection of EP4 agonists in rats by Mebane et al. revealed significant touch-evoked allodynia, as well as mechanical and thermal hyperalgesia. ${ }^{29}$ However, St-Jacques et al. later demonstrated a more peripheral EP4 effect localized to the dorsal root ganglion (DRG) neurons, suggesting that $\mathrm{PGE}_{2}$ sensitizes DRG neurons and hereby induces $\mathrm{COX}-2 / \mathrm{PGE}_{2} / \mathrm{EP} 4$ signaling trough EP4 externalization in DRG neurons. ${ }^{30}$

\section{From bench to bedside: translating animal findings to human pain}

The literature reviewed so far shows good evidence that spinal prostaglandins, particularly $\mathrm{PGE}_{2}$, are involved in spinal nociception and sensitization. In summary, COX-1 and COX-2 are upregulated by painful stimuli, spinal $\mathrm{PGE}_{2}$ causes pain and hyperalgesia, and these phenomena are attenuated by spinal application of
COX-inhibitors or EP-antagonists. Unfortunately, the large amount of animal literature is paralleled by only a handful of human studies. Methodological limitations and ethical considerations are probably the main reasons for the limited data. Nevertheless, there is some evidence indicating similar effects in humans. Buvanendran et al. have investigated the relationship between postoperative pain in humans and prostaglandins in the cerebrospinal fluid. They demonstrated that IL- 6 and PGE $_{2}$ increased markedly after total hip replacement. Moreover, the increase in $\mathrm{PGE}_{2}$ was positively correlated to the intensity of postoperative pain, and preoperative administration of the COX-2 inhibitor rofecoxib was able to block this surgery-associated increase in $\mathrm{PGE}_{2} \cdot 31$

\section{Intrathecal NSAIDs in human experimental pain}

Because differentiation between central and peripheral effects of NSAIDs is difficult in humans, most human experimental studies have used intrathecal (i.t.) administration of ketorolac, as this was the only preservative-free preparation with regulatory approval for intrathecal use.

Eisenach et al. ${ }^{32}$ administered $2 \mathrm{mg}$ of ketorolac i.t. in healthy volunteers who underwent experimental pain testing, using the skin sensitization model by topical capsaicin or the ultra-violet $B$ (UV-B) burn model. Hypersensitivity was reduced after i.t. ketorolac only in the UV-B, but not in the capsaicin model. As the UV-B model is considered a model of inflammatory pain, these findings suggest NSAIDs exert central effects mainly in the presence of peripheral inflammation. Conversely, an intense C-fiber stimulation, as produced by the capsaicin model and possibly reflecting neuropathic pain hypersensitivity, does not seem to respond to centrally-administered NSAIDs.

In a study by Arendt-Nielsen et al., 33 the COX-2 inhibitor etoricoxib significantly reduced both local and spreading sensitization, as well as temporal summation, in patients with knee osteoarthritis. Since temporal summation is likely a hypersensitivity phenomenon of dorsal horn neurons, the authors conclude that etoricoxib exerts at least parts of its effect in the central nervous 


\section{COPYRIGHT $^{\odot} 2018$ EDIZIONI MINERVA MEDICA}

system. One could hypothesize that central effects of NSAIDs occur in the presence of a state of peripheral inflammation, which sensitizes dorsal horn neurons by continuous C-fiber nociceptive barrage. This might be the case in osteoarthritis or after UV-burn, in line with the aforementioned effects observed by Eisenach et al. ${ }^{32}$

\section{Intrathecal NSAIDs \\ in the surgical setting}

Most studies investigating i.t. ketorolac in humans were performed in an orthopedic surgical setting, on patients undergoing spinal anesthesia. Compared to animal data, their results are somewhat ambiguous. Lauretti et al. added i.t. morphine, i.t. ketorolac, combined i.t. morphine and ketorolac or placebo to a routine spinal anesthesia (using $15 \mathrm{mg}$ of bupivacaine) for knee arthroplasty in a four-arm double-blinded trial. ${ }^{34}$ Their primary outcome measure was time to first analgesic rescue medication in the post-anesthesia care unit (PACU). Both i.t. morphine and i.t. ketorolac prolonged the time to first rescue medication to 7-8 hours compared to three hours in the placebo group. Co-administration of i.t. morphine and ketorolac resulted in significant potentiation of these effects and prolonged the time to first rescue medication to 16 hours. Furthermore, the combined i.t. morphine and ketorolac group had a significantly lower total analgesic consumption and did not require intravenous opioids at all. On the other hand, in a sample of 30 patients undergoing vaginal hysterectomy under spinal anesthesia with $15 \mathrm{mg}$ bupivacaine and either $2 \mathrm{mg}$ i.t. ketorolac or $2 \mathrm{~mL}$ saline, there was no difference in time to first i.v. morphine dose, postoperative pain scores or total amount of morphine consumed in the PACU. 35

These findings are similar to those by Wang et $a l ., 36$ who tested i.t. ketorolac vs. placebo as an adjunct to spinal anesthesia for hip arthroplasty. Neither post-operative opioid use nor pain scores differed between the two groups. However, the study had to be terminated early because the only ketorolac preparation approved for intrathecal use was no longer manufactured. Malmberg and Yaksh $^{2}$ had shown initially that spinal upregulation of COX and prostaglandins is mediated by continuous afferent $\mathrm{C}$-fiber nociceptive input. As spinal anesthesia blocks this continuous nociceptive input, it might not be a suitable model for detecting central effects of NSAIDs.

\section{Intrathecal NSAIDs in human chronic pain}

The first attempt to use spinal NSAIDs in chronic pain dates 1987, when Pellerin et al. injected acetylsalicylate epidurally in advanced cancer patients. They reported significant and long-lasting analgesia in a series of 60 patients. ${ }^{37}$ Only decades later, Eisenach et al. examined a group of chronic pain patients receiving intrathecal morphine via an implanted pump. ${ }^{35}$ Their usual drug was removed from the pump and replaced by ketorolac or placebo in a double-blinded fashion. They reported a significant analgesic effect, which, however, did not differ between placebo and ketorolac. The amount of $\mathrm{PGE}_{2}$ in the CSF samples was reduced by ketorolac, but only in patients who had high baseline $\mathrm{PGE}_{2}$ concentrations. In patients with normal baseline $\mathrm{PGE}_{2}$, its concentration was not affected by ketorolac. Interestingly, only those patients with high baseline $\mathrm{PGE}_{2}$ and concomitant reduction by ketorolac reported a strong analgesic effect, partly in line with animal data.

\section{Conclusions}

The central effects of NSAIDs are supported by a large body of evidence in animals. The central effects in inflammatory pain are robustly explained, whereby spinal inflammation-induced COX-2 expression and local $\mathrm{PGE}_{2}$ concentration increases in the dorsal horn are linked to a decreased efficacy of inhibitory glycinergic interneurons. The importance of glycine in human pain modulation could be recently confirmed. ${ }^{38}$ Neuropathic pain seems to be linked to mechanisms largely independent of the COX-2-PGE EP2 pathway, as demonstrated in multiple animal models. ${ }^{39}$

Methodological restrictions in humans, such as risks associated with repeated dural punctures, difficulty to study inhibitory postsynaptic currents and inflammatory mediators the same way as in animals, as well as ethical considerations, 


\section{COPYRIGHT $^{\circ} 2018$ EDIZIONI MINERVA MEDICA}

CENTRAL ANALGESIC EFFECTS OF NSAIDS

VUILLEUMIER

make it difficult to selectively investigate central effects of NSAIDs. Human research must rely on surrogate markers and assess indirect effects. Results are therefore less clear-cut as compared to animal studies. Future research might address these questions using experimental settings other than spinal anesthesia with intrathecal NSAIDs. General anesthesia or measures of spinal hyperexcitability (e.g. nociceptive reflexes or temporal summation) might provide more insight. Translational research has nevertheless produced significant results since the first description of NSAID's spinal effects by Malmberg et al. ${ }^{2}$ and has increased our knowledge on the central prostaglandin $E_{2}$ signaling pathway in inflammatory pain. Moreover, specific EP-antagonists might offer a novel approach to pain treatment, although their use is currently confined to the laboratory setting.

\section{Key messages}

- Animal data revealed significant increases in spinal cord $\mathrm{PGE}_{2}$ levels in peripheral inflammation as well as in surgical and neuropathic pain models.

- $\mathrm{PGE}_{2}$-induced central sensitization seems to be mediated by a COX-2-PGE 2 response to proinflammatory cytokines, resulting in phosphorylation and inhibition of the glycine receptor $\alpha 3$ in the superficial spinal cord dorsal horn.

- In humans spinal $\mathrm{PGE}_{2}$ build-up is positively correlated to the intensity of postoperative pain, whereby preoperative administration of the COX-2 inhibitors blocks this surgery-associated increase in $\mathrm{PGE}_{2}$.

\section{References}

1. Cryer B, Barnett MA, Wagner J, Wilcox CM. Overuse and Misperceptions of Nonsteroidal Anti-inflammatory Drugs in the United States. Am J Med Sci 2016;352:472-80.

2. Malmberg AB, Yaksh TL. Hyperalgesia mediated by spinal glutamate or substance P receptor blocked by spinal cyclooxygenase inhibition. Science 1992;257:1276-9.

3. Malmberg AB, Yaksh TL. Antinociceptive actions of spinal nonsteroidal anti-inflammatory agents on the formalin test in the rat. J Pharmacol Exp Ther 1992;263:136-46.

4. Seibert K, Zhang Y, Leahy K, Hauser S, Masferrer J, Perkins $\mathrm{W}$, et al. Pharmacological and biochemical demonstra- tion of the role of cyclooxygenase 2 in inflammation and pain. Proc Natl Acad Sci USA 1994;91:12013-7.

5. Vanegas H, Schaible HG. Prostaglandins and cyclooxygenases [correction of cycloxygenases] in the spinal cord. Prog Neurobiol 2001;64:327-63.

6. Natura G, Bär KJ, Eitner A, Boettger MK, Richter F, Hensellek S, et al. Neuronal prostaglandin E2 receptor subtype EP3 mediates antinociception during inflammation. Proc Natl Acad Sci USA 2013;110:13648-53.

7. Coleman RA, Smith WL, Narumiya S. International Union of Pharmacology classification of prostanoid receptors: properties, distribution, and structure of the receptors and their subtypes. Pharmacol Rev 1994;46:205-29.

8. Narumiya S, Sugimoto Y, Ushikubi F. Prostanoid receptors: structures, properties, and functions. Physiol Rev 1999; 79:1193-226.

9. Vane JR, Bakhle YS, Botting RM. Cyclooxygenases 1 and 2. Annu Rev Pharmacol Toxicol 1998;38:97-120.

10. Ferreira SH, Lorenzetti BB. Intrathecal administration of prostaglandin E2 causes sensitization of the primary afferent neuron via the spinal release of glutamate. Inflamm Res 1996;45:499-502.

11. Reinold H, Ahmadi S, Depner UB, Layh B, Heindl C, Hamza M, et al. Spinal inflammatory hyperalgesia is mediated by prostaglandin E receptors of the EP2 subtype. J Clin Invest 2005;115:673-9.

12. Dirig DM, Yaksh TL. Spinal synthesis and release of prostanoids after peripheral injury and inflammation. Adv Exp Med Biol 1999;469:401-8.

13. Kras JV, Dong L, Winkelstein BA. Increased interleukin$1 \alpha$ and prostaglandin E2 expression in the spinal cord at 1 day after painful facet joint injury: evidence of early spinal inflammation. Spine 2014;39:207-12.

14. Hefferan MP, Carter P, Haley M, Loomis CW. Spinal nerve injury activates prostaglandin synthesis in the spinal cord that contributes to early maintenance of tactile allodynia. Pain 2003;101:139-47.

15. Hay CH, Trevethick MA, Wheeldon A, Bowers JS, de Belleroche JS. The potential role of spinal cord cyclooxygenase-2 in the development of Freund's complete adjuvantinduced changes in hyperalgesia and allodynia. Neuroscience 1997; 78:843-50.

16. Samad TA, Moore KA, Sapirstein A, Billet S, Allchorne $\mathrm{A}$, Poole $\mathrm{S}$, et al. Interleukin-1beta-mediated induction of Cox-2 in the CNS contributes to inflammatory pain hypersensitivity. Nature 2001;410:471-5.

17. Zhu X, Conklin DR, Eisenach JC. Preoperative inhibition of cyclooxygenase- 1 in the spinal cord reduces postoperative pain. Anesth Analg 2005;100:1390-3.

18. Shi L, Smolders I, Umbrain V, Lauwers MH, Sarre S, Michotte Y, et al. Peripheral inflammation modifies the effect of intrathecal IL-1beta on spinal PGE2 production mainly through cyclooxygenase-2 activity. A spinal microdialysis study in freely moving rats. Pain 2006;120:307-14.

19. You HJ, Mørch CD, Chen J, Arendt-Nielsen L. Differential antinociceptive effects induced by a selective cyclooxygenase-2 inhibitor (SC-236) on dorsal horn neurons and spinal withdrawal reflexes in anesthetized spinal rats. Neuroscience 2003;121:459-72.

20. Tonai $T$, Taketani $Y$, Ueda $N$, Nishisho $T$, Ohmoto $Y$, Sakata Y, et al. Possible involvement of interleukin-1 in cyclooxygenase- 2 induction after spinal cord injury in rats. J Neurochem 1999;72:302-9.

21. Inoue A, Ikoma $\mathrm{K}$, Morioka N, Kumagai $\mathrm{K}$, Hashimoto $\mathrm{T}$, Hide I, et al. Interleukin-1beta induces substance P release 


\section{COPYRIGHT $^{\odot} 2018$ EDIZIONI MINERVA MEDICA}

VUILLEUMIER

CENTRAL ANALGESIC EFFECTS OF NSAIDS

from primary afferent neurons through the cyclooxygenase- 2 system. J Neurochem 1999;73:2206-13.

22. Beiche F, Scheuerer S, Brune K, Geisslinger G, GoppeltStruebe M. Up-regulation of cyclooxygenase-2 mRNA in the rat spinal cord following peripheral inflammation. FEBS Lett 1996;390:165-9.

23. Ahmadi S, Lippross S, Neuhuber WL, Zeilhofer HU. PGE(2) selectively blocks inhibitory glycinergic neurotransmission onto rat superficial dorsal horn neurons. Nat Neurosci 2002;5:34-40.

24. Harvey RJ, Depner UB, Wässle H, Ahmadi S, Heindl C, Reinold H, et al. GlyR alpha3: an essential target for spinal PGE2-mediated inflammatory pain sensitization. Science 2004;304:884-7.

25. Acuña MA, Yévenes GE, Ralvenius WT, Benke D, Di Lio A, Lara CO, et al. Phosphorylation state-dependent modulation of spinal glycine receptors alleviates inflammatory pain. J Clin Invest 2016;126:2547-60.

26. Oliva P, Berrino L, de Novellis V, Palazzo E, Marabese I, Siniscalco D, et al. Role of periaqueductal grey prostaglandin receptors in formalin-induced hyperalgesia. Eur J Pharmacol 2006;530:40-7.

27. Nakayama Y, Omote K, Kawamata T, Namiki A. Role of prostaglandin receptor subtype EP1 in prostaglandin E2induced nociceptive transmission in the rat spinal dorsal horn. Brain Res 2004;1010:62-8.

28. Johansson T, Narumiya S, Zeilhofer HU. Contribution of peripheral versus central EP1 prostaglandin receptors to inflammatory pain. Neurosci Lett 2011;495:98-101.

29. Mebane H, Turnbach ME, Randich A. Spinal EP receptors mediating prostaglandin E2-induced mechanical hyperalgesia, thermal hyperalgesia, and touch-evoked allodynia in rats. J Pain 2003;4:392-9.

30. St-Jacques B, Ma W. Prostaglandin E2/EP4 signalling facilitates EP4 receptor externalization in primary sensory neurons in vitro and in vivo. Pain 2013;154:313-23.
31. Buvanendran A, Kroin JS, Berger RA, Hallab NJ, Saha $\mathrm{C}$, Negrescu C, et al. Upregulation of prostaglandin E2 and interleukins in the central nervous system and peripheral tissue during and after surgery in humans. Anesthesiology 2006;104:403-10.

32. Eisenach JC, Curry R, Tong C, Houle TT, Yaksh TL. Effects of intrathecal ketorolac on human experimental pain. Anesthesiology 2010;112:1216-24.

33. Arendt-Nielsen L, Egsgaard LL, Petersen KK. Evidence for a central mode of action for etoricoxib (COX-2 inhibitor) in patients with painful knee osteoarthritis. Pain 2016;157:1634-44.

34. Lauretti GR, Righeti CC, Mattos AL. Intrathecal ketorolac enhances intrathecal morphine analgesia following total knee arthroplasty. J Anaesthesiol Clin Pharmacol 2013;29:503-8.

35. Eisenach JC, Curry R, Rauck R, Pan P, Yaksh TL. Role of spinal cyclooxygenase in human postoperative and chronic pain. Anesthesiology 2010;112:1225-33.

36. Wang L, Bauer M, Curry R, Larsson A, Sessler DI, Eisenach JC. Intrathecal ketorolac does not improve acute or chronic pain after hip arthroplasty: a randomized controlled trial. J Anesth 2014;28:790-3.

37. Pellerin M, Hardy F, Abergel A, Boule D, Palacci JH, Babinet $\mathrm{P}$, et al. [Chronic refractory pain in cancer patients. Value of the spinal injection of lysine acetylsalicylate. 60 cases]. Presse Med 1987;16:1465-8. French.

38. Vuilleumier PH, Fritsche R, Schliessbach J, Schmitt B, Arendt-Nielsen L, Zeilhofer HU, et al. Mutations affecting glycinergic neurotransmission in hyperekplexia increase pain sensitivity. Brain 2018;141:63-71.

39. Hösl K, Reinold H, Harvey RJ, Müller U, Narumiya S, Zeilhofer HU. Spinal prostaglandin E receptors of the EP2 subtype and the glycine receptor alpha3 subunit, which mediate central inflammatory hyperalgesia, do not contribute to pain after peripheral nerve injury or formalin injection. Pain 2006;126:46-53.

Conflicts of interest.-The authors certify that there is no conflict of interest with any financial organization regarding the material discussed in the manuscript.

Article first published online: May 9, 2018. - Manuscript accepted: May 3, 2018. - Manuscript revised: April 3, 2018. - Manuscript received: November 30, 2017. 